colon from one-half to one pint of warm olive oil or cottonseed oil, to be retained over night or as long as possible, a towel being placed over the anus and a rubber sheet on the bed as protection in case of leakage. This injection is often followed by the passage, a few hours later, of the oil and an abundance of mucus, with disappearance of the colic and no recurrence for a long time, if at all.

57 West Fifty-Eighth Street.

\section{METHODS OF ADMINISTERING SAI.INE AND OTHER SOLUTIONS TO INFANTS AND CHILDREN *}

\author{
JOHN AIKMAN, M.D. \\ ROCHESTER, N. Y
}

The administration of physiologic sodium chloricl and other solutions to replace fluids lost from the body is a valuable method of carrying certain cases over critical periods. Loss of fluid is more serious in children because of the vomiting that so often accompanies grave illness, and because of the difficulty of giving water by mouth. A relative acidosis may easily result, which in a few hours may greatly increase the gravity of the attack. The reduction of fluid is marked in cases with a history of numerous watery stools, characterized by a rapid loss of weight, hollow eyes, and drawn, pinched expression of the facies. It is my object here to present an outline of the several methods of administering fluids, and to discuss their relative merits.

The method of greatest service is that which will permit the introduction and retention of large amounts of fluid with the greatest ease to the operator and with the least danger and discomfort to the patient.

\section{OUTLINE OF METIIODS}

1. Administration of Fluid by Mouth.-If a child can take and retain sufficient quantities by mouth, it is obvious that no other method is necessary, except for special indications, such as a great loss of blood as the result of hemorrhage.

2. Rectal Administration. - The introduction of fluids by rectum, either through enemas, return flow tubes, or by the drip method has been employed for some years with satisfactory results. If the fluid is properly introduced, there is no doubt that considerable absorption takes place. The method is objectionable in children, because of factors not encountered in adults. Unless the child is prostrated, he is apt to be restless, making the retention of the tubes very difficult. It takes considerable time to introduce the necessary amount of fluid, and the child is more or less disturbed thereby, which interferes with his rest. The fluid is apt to be promptly expelled, especially in diarrhea, and there is always some doubt as to the amount which has been absorbed. However, a great variety of fluids may be given by this method, and there is no special danger attached to the procedure.

3. Hypodermoclysis.-In children, especially very small children, small amounts of fluid can be quickly given by this method through a Luer syringe and an intravenots needle, or it can be given by gravity. We have often given fluid in several different areas at

* Read before the Rochester Pathological Society, Nov. 19, 1919. the same time. The procedure is very painful and causes restlessness, especially in older children. The amount that can be given is quite limited. While absorption is slow it is sure. It is safest to use physiologic sodium chlorid solution, although I once used a fresh solution of 1 per cent. sodium bicarbonate in two children over 5 years of age who were desperately ill with ileocolitis accompanied by vomiting and very frequent stools. No trouble resulted from repeated injection of fluid, and both children made an uneventful recovery; there is always danger that solutions of sodium bicarbonate will change in character and cause a slough. These cases were seen several years ago; under similar conditions today I would give physiologic sodium chlorid solution by another method that would better answer the indications and cause much less pain.

4. Intravenous Injection.-It is not necessary to mention all the fluids that may be given by this method. It is estimated that an amount equal to one sixtieth of the body weight can be introduced through the veins, but much more can be used if the loss of fluid has been extreme. Excretion of the fluid begins at once; if prolonged results are to be obtained, it is often necessary to give repeated infusions. The limited amount that may be given and the difficulty of entering the vein of a small child are the chief objections to this method. With proper technic the method is safe, but it will be acknowledged by any one who has tried it that it is difficult to make an intravenous injection on a small, active infant, especially into the veins at the elbow. In order to overcome this difficulty, other methods have been devised.

(a) Intrasinus Injection: In an infant with open fontanel this offers the best means of introducing fluid into the blood stream. The method was first studied by Tobler and was introduced in this country only a few years ago by Helmholz. By this technic, the fluid can be injected through the anterior fontanel directly into the superior longitudinal sinus. As the sinus lies from 2 to 5 millimeters from the skin, it can be easily entered if the fontanel is not closed; at the posterior angle of the fontanel the sinus is wider and deeper. The child is held prone on the table by an assistant, while the needle is introduced in the median line just in front of the posterior angle. If the child is quiet, it is very easy to withdraw blood or to introduce fluid; by means of a Luer syringe, rubber tubing and a threeway cock any amount of fluid can be given without removing the syringe. The needle should be short, and the long point usually found on intravenous needles should be filed away. If a glass syringe is attached before introduction of the needle, constant suction may be maintained for the purpose of discerning when the sinus is entered. If negative pressure is. not produced, blood will not flow so quickly, while the operator may push the needle through the inferior wall of the sinus, blood flowing only when the needle is withdrawn. This accident may also be avoided if the needle be introduced at an angle, directed backward.

Any solution adapted to intravenous administration can be given in this way; with physiologic sodium chlorid, glucose and other mild solutions there is practically no danger. It is also an excellent method for transfusion of citrated whole blood in infants.

In cases with a closed fontanel, the external jugular vein can often be used successfully.

(b) Injection in the Femoral Vein: This method is practicel in this city by several physicians working 
in the venereal clinics and has produced satisfactory results, especially in children. Arsphenamin may be given in this way to very small infants, and although repeated injections have been made on the same child, no serious complications and no cases of thrombosis have been reported.

While it is possible that the method was previously in use by others, it was introduced in this city some years ago by Dr. E. T. Wentworth, who elaborated the following technic, which has since been followed by himself and others:

If the injection is to be made into the right femoral vein, the operator should stand to the right side of the patient, and with the fingers of the left hand palpate the femoral artery as it passes under Poupart's ligament midway between the anterior superior spine of the ilium and the symphysis pubis. The fingers cover the artery while a medium-sized intravenous needle is introduced into the vein just to the left of the finger tips. The needle may be pushed straight in or at an angle directed upward and backward. A syringe is attached to the needle, and constant suction is maintained until a flow of blood shows that the vein has been entered; the syringe can then be removed and the fluid introduced by gravity. If a Luer-Kaufman syringe with a glass offset is used, the solution can be introduced immediately through the syringe itself.

By this method the intravenous administration to children of any age is greatly simplified; if arsphenamin can be given with so little danger, then certainly saline, glucose and other mild solutions can safely be used.

These modifications make the intravenous introduction of fluid comparatively easy; the child is disturbed only little, and a definite amoutat of solution may be given quickly, although the amount that can be given at one time is limited.

5. Intraperitoneal Injection.-This was first used in St. Bartholomew's Hospital and was introduced in this country by Howland. Blackfan and Maxcy have reported the successful employment of this method.

The instruments needed are a medium-sized intravenous needle, an infusion bottle and rubber tubing. The skin of the abdomen is carefully sterilized with tincture of iodin and alcohol. The skin and stbcutaneous tissue are picked up between the thumb and forefinger, and the needle is introduced in an upward direction through the abdominal wall in the midline just below the umbilicus. Care must be taken to avoid piercing a distended bladder, and while there is also danger of puncturing the intestine, no record of this accident has come to my attention. In cases in which necropsy was performed there was found a small hemorrhagic area in the abdominal wall and peritoneum, but no injury of serious importance.

When the needle has passed into the peritoneal cavity, the solution is introduced by gravity. At first I used a Luer syringe; but later I found it much easier to employ the infusion bottle. I have always used warm physiologic sodium chlorid solution, of which from 100 to 250 c.c.., in older children from 300 to 400 c.c., may be given every twelve to twenty-four hours, in fact, if no untoward signs develop, fluid may be given until the abdomen becomes slightly distended. However, the injection must be made slowly in all cases, and overdistention of the abdomen must be avoided. After the operation, the abdomen is covered with a sterile dressing. It has been shown by the phenolsulphonephthalein test and by necropsy that from 40 to 60 per cent. of the fluid is absorbed in one hour. The remaining solution acts as a reserve, the gradual absorption of which explains the more protracted improvement as compared to results obtained by other methods.

We had used the other methods at the Infants Summer Hospital, but this year we chose the intraperitoneal route for children who had lost large amounts of fluid by vomiting and diarrhea. It proved superior to all other methods because of the ease and rapidity of administration, the volume of fluid that can be given at one time, and the certainty that no fluid will be lost. The results from this treatment are remarkable; and although it has been used only in the most serious cases, the results have been most satisfactory.

The following case is reported to show that repeated injections may be made without injury to the intestine:

Rita C., aged 1 year, weighing 11/4 pounds, was admitted to the Infants Summer Hospital, August 18, with a history of vomiting and diarrhea for the previous ten days. She was born at eight months, the delivery being instrumental; the birth weight was $73 / 4$ pounds. The child had been breast fed until the present illness when she was put on condensed milk.

On admission the child had a wasted appearance and was semiconscious. The physical examination revealed slight stomatitis, coldness of the extremities, weakness, and great loss of water from the tissues; the temperature was $101.6 \mathrm{~F}$; the pulse, 148. Stimulating treatment was at once instituted. On the first day she retained onc-half ounce of rice water, vomiting all other fluids; on the second day seven stools were passed and very little fluid was retained. The feces contained blood and mucus. There was no improvement in the bowel condition on the third day, and the loss of fluid was producing great prostration. Since no results followed administration of fluid by rectum or the use of stimulants hypodermically, 100 c.c. of physiologic sodium chlorid solution were injected intraperitoneally, with favorable results. It was not until the thirty-fifth day of the disease that the child passed normal stools; even then the bowel condition was far from satisfac. tory. During this time there had been an average of seven mucous and bloody stools daily; in fact, many of the movements were thick mucus stained with blood. For days in succession she would take less than 1 ounce at a feeding, and vomited a great part of all fluid ingested. She failed to such an extent that it did not seem possible that she could live another day. The temperature ranged from 99 to 102.6, most of the time above $101 \mathrm{~F}$. In all, ten injections of saline solution were made through the abdominal wall, ranging in quantity from 100 to 250 c.c., six being over 200 c.c.

The fluid carried the child over the critical days until the bowel condition began to iniprove. We had never before seen a child recover after so long and severe an illness. The recovery is evidence of the value of this method of treatment and of the safety with which repeated injections may be made throigh the abdominal wall.

\section{SUM MARY}

1. The administration of saline and other solutions is frequently of great value in carrying children over critical periods of illness, especially in diarrheas with marked loss of fluid.

2. When it is impossible to give fluid by mouth or rectum, intravenous or intra-abdominal injections are indicated. If puncture of the superficial veins proves difficult, the superior longitudinal sinus or the femoral vein may be selected.

184 Alexander Street.

Sanitation in Universities,-Environment plays an important rôle in disease causation and prevention. This is especially true of communicable diseases. It is most important to know and to regulate, so far as possible, the conditions under which students live, the food and water ingested, the air breathed, etc. The division of sanitation is therefore no minor part of a university health service, and ample provisions must be made for this branch of the work. The environment of the student, both on and off the campus, must be regulated and made as sanitary as possible.--John Sundwall, Pub. Hcalth Rep., Nov. 7, 1919. 